\title{
Estudio comparativo del bienestar organizacional durante la pandemia
}

\section{Comparative study of organizational well-being during the pandemic}

\author{
OLVERA-ESPINOSA, Edgar ${ }^{*}$, VILLASEÑOR-PADILLA, Dania Elba y PEDRONI-LARA, \\ Fernando
}

Universidad Tecnológica del Valle de Toluca, México.

ID $1^{\text {er }}$ Autor: Edgar, Olvera-Espinosa / ORC ID: 0000-0001-8872-6575, CVU CONACYT ID: 240003

ID $1^{\text {er }}$ Coautor: Dania Elba, Villaseñor-Padilla / ORC ID: 0000-0002-3003-061X

ID 2 ${ }^{\text {do }}$ Coautor: Fernando, Pedroni-Lara / ORC ID: 0000-0002-9194-4079

DOI: $10.35429 / J P D .2020 .12 .4 .1 .8$

Recibido 10 de Julio, 2020; Aceptado 30 de Diciembre, 2020

\begin{abstract}
Resumen
La presente investigación tiene por objetivo analizar el cambio en el bienestar organizacional, a causa del confinamiento voluntario durante la pandemia por el COVID-19, mediante un estudio comparativo de tipo correlacional para la identificación de variables clave de mejora. La metodología que se aplicó fue, mediante un muestreo de 497 estudiantes y profesores universitarios del Valle de Toluca, en el cual mediante un formulario electrónico se recolectó la información sobre las emociones positivas que experimentaron antes del confinamiento y a seis meses de permanecer en esa condición. Se calcularon las desviaciones estándar para cada variable, al representar los resultados mediante una gráfica radial, es posible apreciar el detrimento de cada una, sin embargo, la proporción en que cambian diferenciada llevó a elaborar una matriz de correlación covarianza. El análisis de los resultados, mediante la hoja de cálculo y el formato condicional en la matriz, permite identificar variables clave que la organización puede promover para la mejora del bienestar organizacional.
\end{abstract}

COVID-19, Bienestar organizacional, Emociones positivas

\begin{abstract}
The objective of this research is to analyze the change in organizational well-being, due to voluntary confinement during the COVID-19 pandemic, through a correlational comparative study to identify key variables for improvement. The methodology that was applied was, through a sample of 504 students and university professors from the Toluca Valley, in which, through an electronic form, information was collected on the positive emotions they experienced before confinement and six months after remaining in that condition. The standard deviations were calculated for each variable, when representing the results by means of a radial graph, it is possible to appreciate the detriment of each one, however the proportion in which they change differentiated led to the elaboration of a correlationcovariance matrix. The analysis of the results, using the spreadsheet and the conditional format in the matrix, allows identifying key variables that the organization can promote to improve organizational well-being.
\end{abstract}

COVID-19, Organizational well-being, Positive emotions

Citación: OLVERA-ESPINOSA, Edgar, VILLASEÑOR-PADILLA, Dania Elba y PEDRONI-LARA, Fernando. Estudio comparativo del bienestar organizacional durante la pandemia. Revista de Didáctica Práctica. 2020. 4-12:1-8.

\footnotetext{
*Correspondencia al Autor (Correo Electrónico: edgar.olvera@utvtol.edu.mx)

$\dagger$ Investigador contribuyendo como primer autor.
} 


\section{Introducción}

El bienestar organizacional como en las personas se evalúa mediante la manifestación de síntomas o variables que se modifican a causa de factores internos e internos. La pandemia ocasionada por el COVID-19 en el ámbito mundial durante 2020, ha modificado las formas de interacción entre las personas y las organizaciones. Las organizaciones surgen como resultado de una necesidad social o del mercado, con el propósito de satisfacer necesidades de las personas (Chiavenato, Idalberto, \& Arao, 2017).

De acuerdo con la Organización Mundial de la Salud, el trabajo beneficia la salud mental y el entorno laboral negativo puede afectar a las personas al grado de causar problemas físicos o psíquicos. La depresión y ansiedad, han tenido repercusiones económicas estimadas anualmente en una pérdida de un billón de dólares, las organizaciones pueden aplicar medidas para mejorar la salud mental en el trabajo, que como consecuencia traen el incremento en la productividad, de 4 dólares por cada dólar invertido en salud organizacional (OMS, 2020).

En esta investigación se realizó un análisis a una muestra de 497 estudiantes y profesores universitarios a razón de 14 estudiantes por profesor, mediante un formulario electrónico que se aplicó en línea para medir la afectación de emociones positivas antes de la pandemia y a seis meses del confinamiento voluntario; mediante un estudio comparativo correlacional se identificó las variables que han sido más afectadas, pero también el comportamiento que tiene una con otra. De los resultados obtenidos es posible identificar las que pueden promover una mejora en el bienestar organizacional.

A diferencia de otras técnicas se evaluó la validez del instrumento mediante el Alfa de Cronbach, se comparó la incidencia mediante la correlación, y se validó mediante la covarianza, y para identificar si la relación entre variables ocurre en la misma dirección.
Las emociones positivas que se midieron son las propuestas en los estudios realizados por Harlen Yadira Alpízar Rojas y Deilin Elena Salas Marín (Alpízar Rojas \& Salas Marín, 2017). El instrumento de medición se compone de escalas de Likert para estandarizar la medición y se proporcionó la definición para precisar la variable a los aplicantes.

El análisis de las variables permite conocer el grado de cambio entre variables, mediante tablas y gráficos, así como precisar sobre las de mayor incidencia.

El principal problema que se presenta es que se percibe una sensación de malestar organizacional a causa del distanciamiento social y se busca mejor el bienestar organizacional. Primeramente, se presenta un acercamiento teórico para comprender el análisis de las emociones positivas, se presenta el planteamiento metodológico mediante el cual se realiza en análisis correlacional de variables $\mathrm{y}$ se presentan las aportaciones de la investigación.

La hipótesis central es: $h$ : El análisis correlacional permite identificar las emociones positivas que sostienen el bienestar organizacional.

\section{Marco Teórico}

\section{Bienestar Organizacional}

El bienestar de las organizaciones es una suma de factores materiales, físicos, estratégicos, pero también psicológicos y humanos, Marisa Salanova lo define como "un bienestar integral, que engloba el bienestar físico, psicológico/mental y social, en una relación recíproca, nutritiva y sostenible a lo largo del tiempo" (Soto, 2020). Por lo que en esta investigación se consideraron las emociones positivas como parte del elemento psicológico/mental, que inciden en la organización. 


\section{La organización saludable}

De acuerdo con estudios realizados (Salanova, 2009), las organizaciones saludables, son resilientes. Ello no significa que no exista problemáticas, sino que los beneficios económicos y sociales superan las organizaciones tóxicas, de modo que la salud laboral puede ser un fin estratégico para cumplir con el propósito organizacional.

La organización que desea permanecer y sobreponerse al cambio social y económico requiere adaptarse en tiempo de crisis, la mejor forma de mantener sus recursos financieros, y económicos es mediante mantener a sus empleados motivados y psicológicamente sanos. La mayoría de los estudios de clima organizacional identifican las debilidades y trabajan sobre ellas. En este sentido, las emociones positivas forman parte del contexto organizacional positivo con el cual la organización puede ser más resiliente mediante esfuerzos sistemáticos, proactivos y buenas prácticas.

\section{Emociones positivas}

Las emociones positivas derivan de la psicología positiva la cual es definida por Martin Selingman como "el estudio científico de las experiencias positivas, los rasgos individuales positivos, las instituciones que facilitan su desarrollo y los programas que ayudan a mejorar la calidad de vida de los individuos, mientras previene la incidencia de la psicopatología" citado por (Alpízar Rojas \& Salas Marín, 2017). De modo que las emociones positivas que se seleccionaron para este estudio son las que a continuación se presentan, y se definen para una interpretación precisa de la medición (Alpízar Rojas \& Salas Marín, 2017):

Optimismo: Se entiende como cuando: Remite a expectativas positivas y objetivos de futuro y cuya relación con variables como la perseverancia, el logro, la salud física y el bienestar han hecho de esta materia uno de los puntos centrales de la psicología positiva.

Resiliencia: Se entiende como la capacidad de una adaptación exitosa, funcional, positiva o competente a pesar de un alto grado de riesgo biológico o psicosocial, o de estrés crónico, o después de un severo o prolongado trauma ( (Contreras, 2006).

ISSN: 2523-2444

ECORFAN ${ }^{\circledR}$ Todos los derechos reservados
Fluidez: Se define como "el estado de experiencia óptima que las personas expresan cuando están intensamente implicadas en lo que están haciendo y que les resulta divertido hacer" (Mesurado, 2009). Implica un estado de consciencia en el cual la persona se incorpora de lleno a una actividad o meta fijada previamente, permanece fijamente involucrada en ella y pasa su tiempo casi sin notarlo

Felicidad / Bienestar: Es el estado que conjuga el bienestar y los sentimientos positivos y que lleva a la persona a sentirse autorrealizada, y a posicionarse con una actitud diferente ante la vida (Alpízar Rojas \& Salas Marín, 2017).

Creatividad: La creatividad es la capacidad de crear, de producir cosas nuevas. Es la capacidad que tiene el cerebro humano para llegar a conclusiones e ideas nuevas y resolver problemas de una forma original (Poseck, 2006).

Humor / Risa: Es la capacidad para reconocer con alegría lo incongruente, para ver la adversidad de una manera benigna y para provocar la risa en los demás o experimentarla uno mismo (Martínez, 2006), es ese punto de escape que permite realizar un alto a todo lo que acontece y estresa, y poder disfrutar de lo que se está viviendo en un determinado momento.

Inteligencia emocional: Es un el tipo de inteligencia social que engloba la habilidad de controlar nuestras propias emociones y las de los demás, así como de discriminar entre ellas y utilizar la información que nos proporciona para guiar nuestro pensamiento y nuestras acciones (Molero, Saiz, \& Martínez, 1998).

Autoeficacia: Se entiende como las "creencias en las propias capacidades para organizar y ejecutar los cursos de acción requeridos que producirán determinados logros o resultados" (Alpízar Rojas \& Salas Marín, 2017). Las creencias que poseemos acerca de nosotros mismos afectan a nuestras conductas, pensamientos y sentimientos en varios sentidos.
OLVERA-ESPINOSA, Edgar, VILLASEÑORPADILLA, Dania Elba y PEDRONI-LARA, Fernando. Estudio comparativo del bienestar organizacional durante la pandemia. Revista de Didáctica Práctica. 2020 


\section{Metodología por desarrollar}

El tamaño de la muestra fue de 497 estudiantes y profesores universitarios a razón de 14 estudiantes por profesor, de la cual $35 \%$ fueron hombres y $65 \%$ mujeres; $92 \%$ fueron estudiantes y $7 \%$ docentes.

Se elaboró un formulario electrónico para medir la afectación de emociones positivas antes de la pandemia y a seis meses del confinamiento voluntario, mismo que se aplicó en línea en una institución de educación superior pública. La escala de actitud que se diseñó fue del 1 al 5 sin escala intermedia, indicando en el instrumento la leyenda: ¿Cómo se ha sentido? Donde 1 representa que no se ha desarrollado esta emoción y 5 que la ha experimentado en plenitud.

Una vez recolectados los datos se validó el instrumento mediante el Alfa de Cronbach:

$\alpha=\frac{K}{K-1}\left[1-\frac{\Sigma s^{2} i}{\Sigma s^{2} t}\right]$

Se calcularon los promedios, la desviación estándar y varianza, por variable. Se calculó la diferencia de los promedios para identificar las variables que tuvieron mayor diferencial a lo largo de los seis meses del confinamiento. Dicha información se tabuló y graficó para comparar los resultados antes y durante la pandemia.

Para realizar el estudio comparativo correlacional se elaboró una matriz de correlación, el coeficiente de correlación se calculó mediante Microsoft Excel® con la función:

$=$ COEF.DE.CORREL $($ datos_y, datos_x)

$\mathrm{Si}$ bien, usualmente forma una escalinata, ya que al cruzar las variables se duplicarían, en el ángulo superior derecho se calculó la covariación entre las variables, también con Microsoft Excel®, con la función:

= COVAR(datos_y, datos_x)

Se procedió al análisis para identificar las aportaciones de esta investigación.

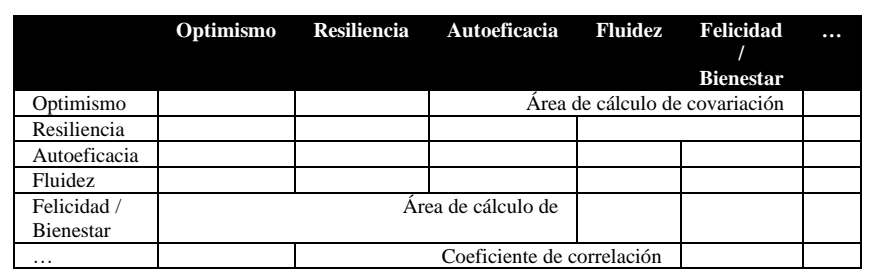

Tabla 1 Descripción de Matriz de coeficiente de correlación y covarianza

Fuente: Elaboración propia

Finalmente, al identificar las variables con mayor incidencia se realizó la suma de coeficientes de correlación de mayor incidencia, por un lado, y por el otro, la suma de la covariación, antes y en este momento, y se obtuvieron datos que permiten apreciar, las emociones positivas en las cuales se puede apoyar la organización para mejorar el bienestar.

Al trazar el gráfico de líneas es posible apreciar los mayores diferenciales.

\section{Resultados}

Derivado de la recolección de datos que se realizó en el mes sexto del confinamiento voluntario se validó en instrumento mediante el Alfa de Cronbach, donde se obtuvo el siguiente resultado:

\begin{tabular}{|l|r|}
\hline \multicolumn{2}{|c|}{ Alfa de Cronbach } \\
\hline $\boldsymbol{\alpha}$ & 1.0 \\
\hline $\mathrm{K}$ & 8 \\
\hline $\mathrm{S}_{\mathrm{i}}$ & 11.8 \\
\hline $\mathrm{S}_{\mathrm{t}}$ & 197.5 \\
\hline
\end{tabular}

Tabla 2 Cálculo del Alfa de Cronbach Fuente: Elaboración propia

Considerando que el $\alpha=1.0, \quad$ se determinó el instrumento como válido. resultado:

El cálculo de los promedios dio como

\begin{tabular}{|l|r|r|}
\hline & Antes & En este momento \\
\hline Optimismo & 3.42 & 2.80 \\
\hline Resiliencia & 3.22 & 2.97 \\
\hline Autoeficacia & 3.46 & 3.07 \\
\hline Fluidez & 3.37 & 2.86 \\
\hline Felicidad / Bienestar & 3.57 & 2.85 \\
\hline Creatividad & 3.37 & 3.02 \\
\hline Humor / Risa & 3.66 & 2.91 \\
\hline Inteligencia emocional & 3.66 & 3.03 \\
\hline
\end{tabular}

Tabla 3 Promedios por variable antes del confinamiento y al momento de la aplicación del instrumento (a los seis meses del confinamiento)

Fuente: Elaboración propia

OLVERA-ESPINOSA, Edgar, VILLASEÑORPADILLA, Dania Elba y PEDRONI-LARA, Fernando. Estudio comparativo del bienestar organizacional durante la pandemia. Revista de Didáctica Práctica. 2020 
Si bien, los valores se observan entre 3.0 y 4.0 de las escalas de Likert antes del confinamiento, a los seis meses del confinamiento se identificaron variables entre 2.0 y 3.0, se calculó la diferencia entre ambos promedios, obteniendo:

\begin{tabular}{|l|r|r|r|}
\hline & Antes & \multicolumn{2}{|c|}{$\begin{array}{c}\text { En este } \\
\text { momento }\end{array}$} \\
\hline Optimismo & 3.42 & 2.80 & 0.61 \\
\hline Resiliencia & 3.22 & 2.97 & 0.25 \\
\hline Autoeficacia & 3.46 & 3.07 & 0.39 \\
\hline Fluidez & 3.37 & 2.86 & 0.51 \\
\hline Felicidad / Bienestar & 3.57 & 2.85 & 0.71 \\
\hline Creatividad & 3.37 & 3.02 & 0.35 \\
\hline Humor / Risa & 3.66 & 2.91 & 0.75 \\
\hline Inteligencia emocional & 3.66 & 3.03 & 0.63 \\
\hline
\end{tabular}

Tabla 4 Diferencia entre las medias antes del confinamiento y al momento de la aplicación del instrumento (a los seis meses del confinamiento)

Fuente: Elaboración propia

En la tabla 4 es posible apreciar que el optimismo, la Felicidad / Bienestar y el Humor / Risa, son las que tienen una mayor diferencia de promedios, por lo que se identifican las áreas de oportunidad que podrían atenderse, se procedió al graficar donde se obtuvo:

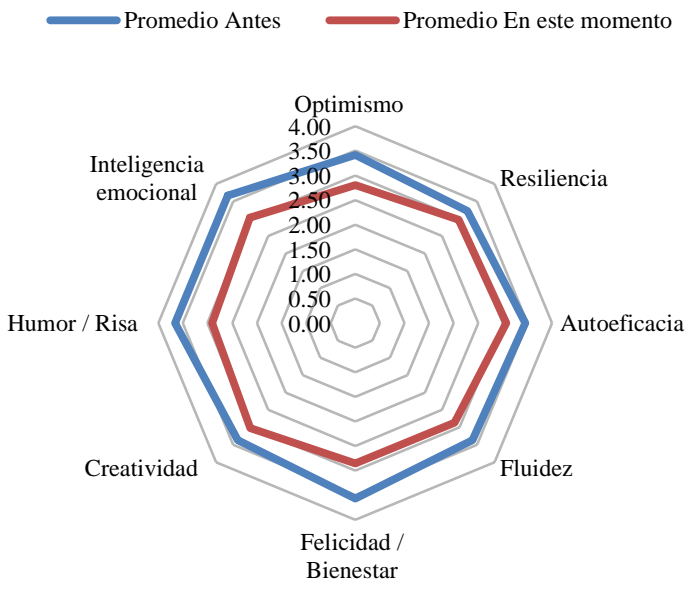

Gráfico 1 Comparativo de los promedios por variable Fuente: Elaboración propia

En el gráfico 1, es posible apreciar esa diferencia, sin embargo, también es posible apreciar que la resiliencia, creatividad y autoeficacia son las que menor diferencia tuvieron. Considerando que la resiliencia es un factor que favorece la salud organizacional, podría determinarse como un factor positivo, para ello se graficó la diferencia de promedios:

\section{Diferencia de promedios antes y en este momento}

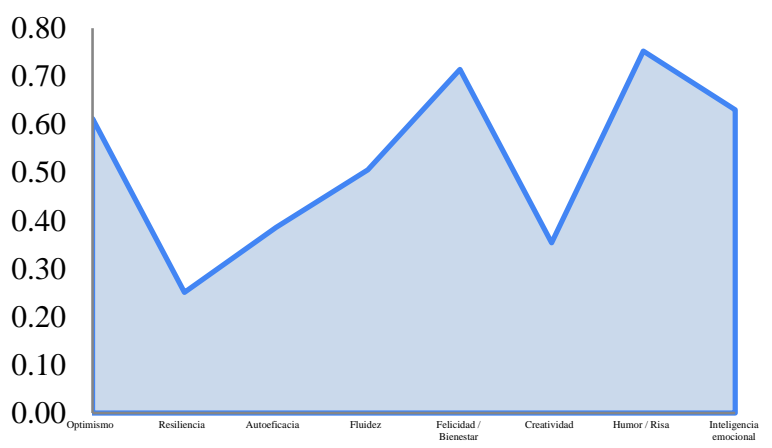

Gráfico 2 Diferencia de promedios antes del confinamiento y al momento de la aplicación del instrumento (a los seis meses del confinamiento)

Fuente: Elaboración propia

De esta manera, en el gráfico 2, se aprecia como además de la resiliencia, la creatividad seguida la autoeficacia, son las que menor diferencia tuvieron.

No obstante, la información que se obtuvo, al calcular el coeficiente de correlación y la covarianza, se validó las variables:

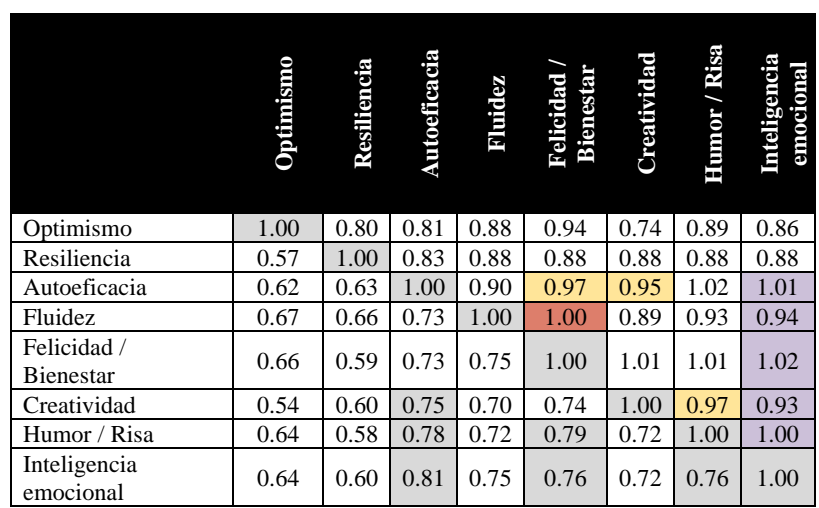

Tabla 5 Coeficiente de correlación y covarianza antes del confinamiento

Fuente: Elaboración propia

Es de destacar, que las variables que se encontraron con un mayor coeficiente correlación (considerando, los valores mayores que 0.75 ) fueron:

\begin{tabular}{|l|l|l|}
\hline Creatividad & \multirow{2}{*}{ Autoeficacia } & 0.75 \\
\cline { 1 - 1 } Humor / Risa & & 0.78 \\
\hline Inteligencia emocional & & $\mathbf{0 . 8 1}$ \\
\hline Humor / Risa & Felicidad / & 0.79 \\
\hline Inteligencia emocional & Bienestar & 0.76 \\
\cline { 3 - 3 } & Humor / Risa & 0.76 \\
\hline
\end{tabular}

Tabla 6 Variables con mayor coeficiente de correlación antes del confinamiento

Fuente: Elaboración propia
OLVERA-ESPINOSA, Edgar, VILLASEÑORPADILLA, Dania Elba y PEDRONI-LARA, Fernando. Estudio comparativo del bienestar organizacional durante la pandemia. Revista de Didáctica Práctica. 2020 
A partir de la tabla 6 , se podría decir que la autoeficacia es la que tiene mayor incidencia de variables por el coeficiente de correlación, antes de la contingencia.

Respecto a la covarianza se observa que en todas las variables es positiva y las aproximaciones a 1.0 son las que presentan mayor dependencia directa, de tal manera que se obtuvo:

\begin{tabular}{|l|l|r|}
\hline \multirow{5}{*}{ Inteligencia emocional } & Fluidez & 1.01 \\
\cline { 2 - 3 } & $\begin{array}{l}\text { Felicidad / } \\
\text { Bienestar }\end{array}$ & 0.94 \\
\cline { 2 - 3 } & Creatividad & 1.02 \\
\cline { 2 - 3 } & $\begin{array}{l}\text { Humor / } \\
\text { Risa }\end{array}$ & 0.93 \\
\cline { 2 - 3 } & Autoeficacia & 1.00 \\
\hline Autoeficacia & $\begin{array}{l}\text { Felicidad / } \\
\text { Bienestar }\end{array}$ & 0.97 \\
\cline { 2 - 3 } & Creatividad & 0.96 \\
\hline Creatividad & $\begin{array}{l}\text { Humor / } \\
\text { Risa }\end{array}$ \\
\hline Felicidad / Bienestar & Fluidez & 0.97 \\
\hline
\end{tabular}

Tabla 7 Variables con mayor covarianza antes del confinamiento

Fuente: Elaboración propia

De la tabla 7, se observa que las variables que tienen mayor covarianza son: Inteligencia Emocional, Creatividad, Felicidad / Bienestar, creatividad y Humor / Risa.

La medición a seis meses del confinamiento arrojó los siguientes resultados:

\begin{tabular}{|c|c|c|c|c|c|c|c|c|}
\hline & 营 & 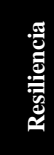 & 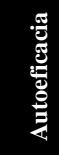 & $\frac{\mathrm{d}}{\mathrm{g}}$ & 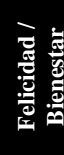 & 宽 & 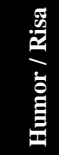 & 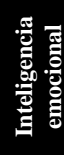 \\
\hline Optim & .00 & 0.74 & 0.71 & 0.72 & 0.94 & 0.71 & 0.78 & 0.74 \\
\hline Resiliencia & .58 & 1.00 & 0.78 & 0.78 & \begin{tabular}{|l|}
0.88 \\
\end{tabular} & 0.68 & 0.77 & 0.79 \\
\hline Autoeficacia & 0.56 & 0.59 & 1.00 & 0.80 & \begin{tabular}{|l|}
0.97 \\
\end{tabular} & 0.96 & 0.99 & 1.05 \\
\hline Fluidez & 0.61 & 0.64 & 0.65 & 1.00 & 1.00 & 0.81 & 0.83 & 0.85 \\
\hline $\begin{array}{l}\text { Felicidad / } \\
\text { Bienestar }\end{array}$ & 0.60 & 0.55 & 0.64 & 0.68 & 1.00 & 0.88 & 1.02 & 0.96 \\
\hline Creatividad & 0.53 & 0.50 & 0.70 & 0.63 & \begin{tabular}{|l|}
0.74 \\
\end{tabular} & 1.00 & 0.99 & 0.91 \\
\hline Humor / Risa & 0.58 & 0.57 & 0.73 & 0.65 & 0.79 & 0.68 & 1.00 & 1.00 \\
\hline $\begin{array}{l}\text { Inteligencia } \\
\text { emocional }\end{array}$ & 0.57 & 0.59 & 0.79 & 0.69 & 0.76 & 0.66 & 0.72 & 1.00 \\
\hline
\end{tabular}

Tabla 8 Coeficiente de correlación y covarianza a seis meses del confinamiento

Fuente: Elaboración propia

Las variables con mayor coeficiente de correlación a seis meses del confinamiento fueron:

\begin{tabular}{|l|l|l|}
\hline Inteligencia emocional & Autoeficacia & 0.79 \\
Felicidad / Bienestar & & 0.76 \\
\cline { 2 - 3 } & Humor / Risa & 0.79 \\
\hline
\end{tabular}

Tabla 9 Variables con mayor coeficiente de correlación a seis meses del confinamiento Fuente: Elaboración propia

La Felicidad / Bienestar tiene la mayor correlación con Autoeficacia y Humor / Risa, seguido de la Inteligencia emocional.

Respecto a la covarianza, a seis meses del confinamiento, se observó que en todas las variables es positiva y las aproximaciones a 1.0 son las que presentan mayor dependencia directa, de tal manera que se obtuvo:

\begin{tabular}{|l|l|r|}
\hline Felicidad / Bienestar & Fluidez & 1.00 \\
& Autoeficacia & 0.97 \\
& & 0.96 \\
\cline { 3 - 3 } Creatividad & 0.99 \\
\cline { 3 - 3 } & & 1.02 \\
\hline Humor / Risa & Felicidad / & 0.96 \\
\hline Inteligencia \\
emocional & Bienestar & 1.05 \\
\cline { 2 - 3 } & Autoeficacia & 1.00 \\
\cline { 2 - 3 } & $\begin{array}{l}\text { Humor } \\
\text { Risa }\end{array}$ \\
\hline
\end{tabular}

Tabla 10 Variables con mayor covarianza a seis meses del confinamiento

Fuente: Elaboración propia

En la tabla 10 se aprecia que las variables que tiene mayor covarianza con más variables son Felicidad / Bienestar, Inteligencia Emocional, seguido de Humor / Risa y autoeficacia.

Al realizar la suma de los coeficientes de correlación de las variables de mayor incidencia se obtuvo:

\begin{tabular}{|l|r|r|}
\hline \multicolumn{1}{|c|}{ Emociones positivas } & \multicolumn{2}{|c|}{$\begin{array}{c}\text { Coeficiente de } \\
\text { correlación } \\
\text { Antes }\end{array}$} \\
& $\begin{array}{c}\text { En este } \\
\text { momento }\end{array}$ \\
\hline Optimismo & 0 & 0 \\
\hline Resiliencia & 0 & 0 \\
\hline Autoeficacia & 0 & 1.55 \\
\hline Fluidez & 0 & 0 \\
\hline Felicidad / Bienestar & 1.56 & 1.56 \\
\hline Creatividad & 0.75 & 0 \\
\hline Humor / Risa & 1.54 & 0.79 \\
\hline Inteligencia emocional & 1.57 & 0.79 \\
\hline
\end{tabular}

Tabla 11 Suma de los Coeficiente de correlación de las variables de mayor incidencia

Fuente: Elaboración propia 
$\mathrm{Si}$ bien antes de la contingencia inteligencia emocional fue en segundo momento de aplicación se redujo la correlación, mientras que autoeficacia cobró relevancia.

La representación gráfica permite apreciar las diferencias, y se observan los coeficientes de correlación predominantes, es decir, las que representan mayor fuerza como emociones positivas, como se presenta en el gráfico 3:

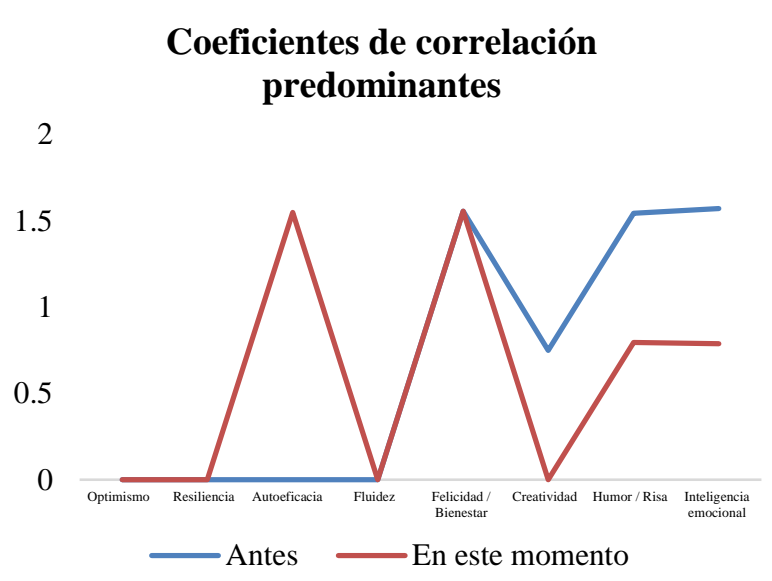

Gráfico 3 Coeficientes de correlación predominantes Fuente: Elaboración propia

De acuerdo con el gráfico 3 Autoeficacia, es una variable que se fortalece al tener una mayor correlación con otras variables, mientras que la de felicidad / Humor permanece igual. Por otra parte, Inteligencia emocional se ve afectada, reduciendo su correlación con otras emociones positivas.

Al comparar las covarianzas fue aún más notable la diferencia

\begin{tabular}{|l|r|r|}
\hline \multicolumn{1}{|c}{ Emociones positivas } & \multicolumn{2}{c|}{ Covarianza } \\
& Antes & En este momento \\
\hline Optimismo & 0 & 0 \\
\hline Resiliencia & 0 & 0 \\
\hline Autoeficacia & 2.98 & 3.97 \\
\hline Fluidez & 1.00 & 1.00 \\
\hline Felicidad / Bienestar & 1.97 & 3.95 \\
\hline Creatividad & 1.94 & 0.96 \\
\hline Humor / Risa & 0.97 & 3.01 \\
\hline Inteligencia emocional & 5.02 & 3.01 \\
\hline
\end{tabular}

Tabla 12 Suma de las covarianzas Fuente: Elaboración propia

En la tabla 12, es de notar que entre ellas, no se encuentran optimismo y resiliencia, que son las que comúnmente se abordan en programas de salud laboral.

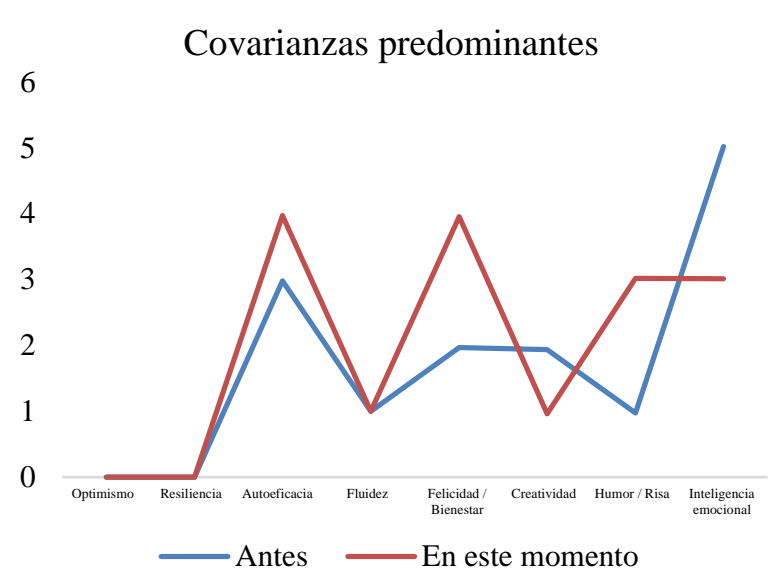

Gráfico 4 Covarianzas predominantes de las variables de mayor incidencia

Fuente: Elaboración propia

En el gráfico 4, se aprecia como la autoeficacia en este momento, supera la covarianza antes de la contingencia y Humor / Risa se encuentra debajo, la fuerza de inteligencia emocional sobre otras variables tuvo una considerable caída, pero Felicidad / Bienestar fue duplicado.

\section{Agradecimiento}

A la Universidad Tecnológica del Valle de Toluca por el patrocinio de esta investigación.

\section{Conclusiones}

El análisis comparativo del bienestar organizacional durante la pandemia, mediante los coeficientes de correlación y covarianza, permiten identificar las variables clave para la mejora del bienestar organizacional. De tal manera de la autoeficacia, que se define como "creencias en las propias capacidades para organizar y ejecutar los cursos de acción requeridos que producirán determinados logros o resultados" (Alpízar Rojas \& Salas Marín, 2017), refiere al reconocimiento de habilidades para ser eficaz en función de las metas, de tal manera que a pesar de que en la media se aprecia una disminución, es una emoción positiva a impulsar, mediante herramientas de organización, para tener claridad sobre los tiempos de cumplimiento de tareas y el conocimiento de planes, programas y proyectos que la organización requiere cumplir. 
La Felicidad / Bienestar: Estado que conjuga el bienestar y los sentimientos positivos y que lleva a la persona a sentirse autorrealizada, y a posicionarse con una actitud diferente ante la vida (Alpízar Rojas \& Salas Marín, 2017), puede llevar a las organizaciones a mejores niveles de autoeficacia

La hipótesis central: El análisis correlacional permite identificar las emociones positivas que sostienen el bienestar organizacional, es aprobada, ya que la covarianza y el coeficiente de correlación, permiten conocer la predominancia de las emociones positivas.

Es posible mejorar la investigación de hacer una comparación de la inclinación de las pendientes de las covarianzas y mediante la realización de una siguiente recolección de datos sobre un siguiente momento, recomendable, al finalizar el confinamiento.

\section{Referencias}

Alpízar Rojas, H. Y., \& Salas Marín, D. E. (2017). El papel de las emociones positivas en el desarrollo de la Psicología Positiva*. Wímb lu, 63-81.

Chiavenato, Idalberto, \& Arao, S. (2017). En Planeación Estratégica (págs. 133-139, 210211). Cidudad de México: Mc Graw Hill Education.

Contreras, F. \&. (2006). Psicología positiva: una nueva perspectiva en psicología. Diversitas-Perspectivas en psicología, 311-319.

Martínez, M. (2006). El estudio científico de las fortalezas trascendentales desde la psicología positiva. Clínica y salud, 245-258.

Mesurado, B. (2009). Comparación de tres modelos teóricos explicativos del constructo experiencia óptima o flow. Interdisciplinaria: Revista de Psicología y Ciencias Afines, 121137.

Molero, C., Saiz, E., \& Martínez, C. (1998). Revisión histórica del concepto de inteligencia: una aproximación a la inteligencia emocional. Revista Latinoamericana de psicología, 11-30.
OMS. (28 de noviembre de 2020). Organziación Mundial de la Salud. Obtenido de Salud Mental: https://www.who.int/mental_health/in_the_wor kplace/es/

Poseck, B. (2006). Psicología positiva: una nueva forma de entender la psicología. Papeles del psicólogo, 3-8.

Salanova, M. (2009). Organizaciones saludables, organizaciones resilientes. DOSSIER: PREVENCIÓN DE RIESGOS LABORALES EN TIEMPOS DE CRISIS, 18-24.

Soto, S. (28 de noviembre de 2020). BUSINESS INSIDER México. Obtenido de Estos son los beneficios del bienestar organizacional, según Marisa Salanova, especialista en psicología organizacional positiva: https://businessinsider.mx/estos-son-losbeneficios-del-bienestar-organizacional-segunmarisa-salanova-especialista-en-psicologiaorganizacional-positiva/ 